\title{
Chemically Assisted Ion Beam Etching of Optical Microresonators
}

\author{
A. Scherer, C.C. Cheng, O. Painter, R. Lee, A. Yariv, J.I. Jewel Y. Fainman
}

1-D, 2-D, 3-D microstructures with lateral dimensions at the scale of the wavelength of light can be used to generate high finesse microresonators. These have been fabricated in the AlGaAs system by combining MBE growth with micro-fabrication. Here we show the applications of anisotropicion beam etching and high resolution lithography in the fabrication of high-finesse micro-resonators and discuss their performance as lowthreshold microlasers, fast optical switches, photonic bandgap mirrors and polarizing beam-splitters. 